\title{
Naturalismo y quietismo
}

\author{
RICHARD RORTY \\ Department of Comparative Literature/Department of Philosophy \\ Stanford University \\ rrorty@stanford.edu
}

Resumen: Brian Leiter divide el mundo filosófico anglosajón en quietistas y
naturalistas. Los primeros ven a la filosofía como un tipo de terapia, que di-
suelve los problemas filosóficos en vez de resolverlos. Los segundos pueden
dividirse, a su vez, siguiendo a Huw Price, en naturalistas del objeto, que
piensan que todo lo que hay es el mundo estudiado por la ciencia, y natura-
listas del sujeto, que sostienen que los humanos somos criaturas naturales y
que la filosofía debe ajustarse a esta perspectiva. Este artículo aboga por un
quietismo sobre algunos problemas en las así llamadas "áreas centrales de la
filosofía", así como por un naturalismo del sujeto. Bajo esta doble perspectiva
se examinan dos controversias filosóficas actuales: la que se da entre McDo-
well y Williamson acerca de lo que puede ser pensado, y la disputa entre
Fodor y Brandom, entre una semántica representacionista y una inferencia-
lista, respectivamente.

Palabras clave: pragmatismo, representacionismo, inferencialismo

\begin{abstract}
Brian Leiter divides the English-speaking philosophical world into quietists and naturalists. The former consider philosophy as a kind of therapy, dissolving philosophical problems rather than solving them. The latter can be divided, according to Huw Price, into object naturalists, who think that all there is is the world studied by science, and subject naturalists, who think that we humans are natural creatures and that philosophy has to adjust itself to this viewpoint. This paper argues for a quietist position about some problems in the so-called "core areas of philosophy", as well as for a subject naturalism. From this dual perspective two current philosophical debates are examined: that between McDowell and Williamson regarding what it is possible to think of; and that between Fodor and Brandom, i.e., between a representationalist and an inferentialist semantics.
\end{abstract}

Key words: pragmatism, representationalism, inferentialism

La filosofía constituye una parte casi invisible de la vida intelectual contemporánea. La mayoría de la gente que no pertenece a los departamentos de filosofía no tiene una idea clara de lo que se supone que los profesores de filosofía le aportan a la cultura. Pocos piensan que valga la pena indagarlo.

La falta de atención que recibe nuestra disciplina se atribuye a veces al tecnicismo de los asuntos que se discuten actualmente; pero ésa no es una buena explicación. Los debates entre los filósofos del lenguaje y de la mente de hoy no son más aburridamente técnicos que aquellos

Diánoia, volumen LI, número 56 (mayo 2006): pp. 3-18. 
que se daban entre los intérpretes y los críticos de Kant en la década de 1790.

El problema no es el estilo en el que se hace actualmente la filosofía en el mundo de habla inglesa; es más bien que muchos de los asuntos discutidos por Descartes, Hume y Kant tuvieron resonancia cultural sólo porque una porción significativa de las clases educadas todavía resistía la secularización de la vida moral y política. ${ }^{1}$ La afirmación de que los seres humanos están solos en el universo y de que no deben buscar ayuda de agentes sobrenaturales iba de la mano con admitir que Demócrito y Epicuro estaban en buena medida en lo correcto acerca de cómo funciona el universo. Los que según el canon son los grandes filósofos modernos realizaron un servicio útil sugiriendo formas de manejar el triunfo del materialismo mecanicista.

Pero a medida que la así llamada "guerra entre la ciencia y la teología" fue decayendo, hubo cada vez menos trabajo útil que pudieran hacer los filósofos. Así como la escolástica medieval se volvió tediosa una vez que la doctrina cristiana se sintetizó con la filosofía griega, así también una buena parte de la filosofía moderna empezó a parecer sin sentido después de que la mayoría de los intelectuales abandonó su fe religiosa o encontró formas de hacerla compatible con la ciencia natural moderna. A pesar de que los agitadores pueden todavía plantear dudas acerca de Darwin entre las masas, los intelectuales -las únicas personas sobre las que los libros de filosofía tienen alguna repercusión-

${ }^{1}$ El cambio más importante producido por la secularización fue el de pensar la moralidad como una cuestión de prohibiciones incondicionales a verla como un intento por encontrar un equilibrio entre necesidades humanas en competencia. Este cambio está bien descrito en el famoso artículo de Elizabeth Anscombe llamado "Modern Moral Philosophy". Ella contrasta prohibiciones severas e incondicionales de cosas tales como el adulterio, la sodomía y el suicidio con el consecuencialismo suave por el que abogaba, como ella dice, "todo filósofo moral académico inglés después [de Sidgwick]". Ese consecuencialismo es, dice Anscombe, "bastante incompatible con la ética judeocristiana" (G.E.M. Anscombe, "Modern Moral Philosophy", p. 34).

En Estados Unidos, actualmente experimentamos un regreso al último tipo de ética: una rebelión de las masas en contra del consecuencialismo de los intelectuales. El enfrentamiento actual entre el estado rojo y el estado azul es un recrudecimiento del viejo conflicto acerca de la secularización de la cultura. Pero, hoy en día, casi nadie se vuelve hacia la filosofía para que ayude en este conflicto. En los siglos XVII y XVIII sí lo hicieron. Escritores como Spinoza y Hume hicieron mucho para promover la causa de la secularización; sin embargo, a lo largo de los siglos XIX y XX se pasó la batuta al arte y a la literatura. Las novelas cuyos personajes discutían conflictos morales sin remitirse a Dios o a la Biblia tomaron el lugar de la filosofía moral.

Diánoia, vol. LI, no. 56 (mayo 2006). 
no tienen esas dudas. No necesitan ni una metafísica sofisticada ni una teoría de la referencia muy elaborada para convencerse de que no hay fantasmas.

Después de que los intelectuales se convencieron de que la ciencia empírica, y no la metafísica, nos dice cómo funcionan las cosas, la filosofía tuvo que elegir entre dos alternativas. Una era seguir la guía de Hegel y convertirse en una combinación de historia intelectual y crítica cultural; el tipo de cosa que nos ofrecieron Heidegger y Dewey, pero también gente como Adorno, Strauss, Arendt, Berlin, Blumenberg y Habermas. Esta forma de hacer filosofía florece sobre todo en el mundo filosófico no anglófono, pero también se encuentra en libros de filósofos estadounidenses como Robert Pippin.

La alternativa fue imitar a Kant y desarrollar un programa de investigación de cubículo, con el fin de ayudar a la filosofía a ganar un lugar en las universidades como una disciplina académica autónoma. Lo que se necesitaba era un programa que se pareciera al de Kant en no darle ningún lugar a la observación, a los experimentos o al conocimiento histórico. Los neokantianos alemanes y los empiristas británicos estaban de acuerdo en que el núcleo de la filosofía era la investigación de algo llamado "Experiencia" o "Conciencia". Frege y Peirce propusieron un programa alternativo que pretende investigar algo llamado "Lenguaje" o "el Signo".

Ambos programas suponían que, tal como la materia puede descomponerse en átomos, lo mismo sucede con la experiencia y el lenguaje. El primer tipo de átomos incluía las ideas simples de Locke, las intuiciones no sintetizadas de Kant, los datos de los sentidos y los objetos de la Wesenschau husserliana. El segundo incluía los sentidos fregeanos, los signos peirceanos y las figuras lingüísticas del Tractatus. Al insistir en que las preguntas que tienen que ver con la relación de átomos inmateriales con partículas físicas estaban en el centro de su disciplina, los filósofos de países anglófonos empujaron hacia la periferia la filosofía social, la historia intelectual, la crítica cultural y a Hegel.

Aun así, siempre ha habido holistas, filósofos que tenían dudas acerca de la existencia de átomos de conciencia o de átomos de significado. Los holistas con frecuencia se convierten en escépticos acerca de la existencia de oscuros sustitutos de la Realidad, como son la "Experiencia", la "Conciencia" y el "Lenguaje". Wittgenstein, el más célebre de estos escépticos, estuvo cerca de sugerir que las así llamadas áreas "centrales" de la filosofía no tienen ninguna función, salvo mantener una disciplina académica en actividad. 
A los escépticos de este tipo se les ha catalogado como "quietistas". Brian Leiter, en su introducción a una compilación publicada recientemente y titulada The Future for Philosophy, divide al mundo filosófico anglófono en "naturalistas" y en "quietistas wittgensteinianos". Estos últimos, dice, ven la filosofía como "un tipo de terapia, que disuelve los problemas filosóficos en vez de resolverlos". ${ }^{2}$ Leiter se alegra al decirnos que éstos se encuentran en minoría, habiéndose impuesto en sólo cuatro departamentos de filosofía importantes (Harvard, Berkeley, Chicago y Pittsburgh). "A diferencia de los wittgensteinianos - escribe Leiter-, los naturalistas creen que los problemas que han preocupado a los filósofos (acerca de la naturaleza de la mente, el conocimiento, la acción, la realidad, la moralidad, etc.) son de hecho reales." 3

Creo que la descripción de Leiter del enfrentamiento entre estos dos campos es bastante exacta. Ha identificado la diferencia de opinión más profunda e insoluble dentro de la filosofía anglófona contemporánea, pero su descripción es engañosa con respecto a un punto. La mayoría de la gente que se pone a sí misma dentro del lado quietista, como yo, dudaría en decir que los problemas que estudian nuestros colegas activistas son irreales. No dividen los problemas filosóficos en reales e ilusorios, sino más bien en aquellos que tienen alguna relevancia para la política cultural y los que no. Los quietistas, por lo menos aquellos que pertenecen a mi secta, piensan que hay que demostrar esa relevancia antes de tomar en serio un problema. Esta perspectiva es un corolario de la máxima de que lo que no importa en la práctica no debe importarle a los filósofos.

Desde este punto de vista, preguntas acerca del lugar de los valores en un mundo de hechos no son más irreales que aquellas sobre cómo la sangre y el vino eucarísticos pueden encarnar la sustancia divina, o acerca de cuántos sacramentos instituyó Jesucristo. Ninguno de estos últimos problemas son problemas para todos, pero su carácter parroquial no los hace ilusorios, pues lo que uno encuentra problemático depende de lo que nos parece importante. Nuestro sentido de la importancia depende en buena medida del vocabulario que empleemos. Así, la política cultural es con frecuencia una pelea entre aquellos que llaman a que evitemos un vocabulario común y aquellos que defienden las viejas formas de hablar.

Consideremos la afirmación de Leiter de que "los neurocientíficos nos hablan del cerebro y los filósofos tratan de averiguar cómo conci-

${ }^{2}$ B. Leiter (comp.), The Future for Philosophy, p. 2.

${ }^{3}$ Ibid., pp. 2-3. 
liar nuestra mejor neurociencia con la capacidad de nuestras mentes de representar cómo es el mundo". ${ }^{4}$ La respuesta quietista consiste en preguntar si realmente queremos conservar el concepto de "representar cómo es el mundo". Tal vez, sugieren, es tiempo de renunciar a la noción de "el mundo", así como a entidades oscuras llamadas "la mente" o "el lenguaje" que contienen representaciones del mundo. El estudio de la historia de la cultura nos ayuda a entender por qué estos conceptos se extendieron, y nos muestra también por qué ciertos conceptos teológicos llegaron a tener la importancia que tuvieron. Pero tal estudio también sugiere que muchas de las ideas centrales de la filosofía moderna, así como muchos temas de teología cristiana, terminan siendo más problemáticos que valiosos.

Philip Pettit, en su contribución a The Future for Philosophy, nos da una descripción de la perspectiva metafilosófica del naturalista un poco más completa que la de Leiter. La filosofía, nos dice, es un intento de reconciliar "la imagen manifiesta de cómo son las cosas" y las "ideas que nos vienen con nuestras prácticas espontáneas cotidianas" con "fidelidad a la imagen intelectual de cómo son las cosas". ${ }^{5}$ En nuestra cultura, dice Pettit, la imagen intelectual es la que nos da la ciencia física. Resume diciendo que:

una imagen naturalista, más o menos mecánica, del universo se nos impone por el desarrollo acumulativo en la física, la biología y la neurociencia, y esto nos reta a buscar dónde puede haber lugar en ese mundo para los fenómenos que siguen tan vivos como siempre en la imagen manifiesta: la conciencia, la libertad, la responsabilidad, el bien, la virtud y otros semejantes. ${ }^{6}$

A pesar de mi veneración por Wilfrid Sellars, que dio origen al discurso acerca de imágenes manifiestas y científicas, quisiera deshacerme de estas metáforas visuales. No deberíamos estar cautivos de la imagen de la imagen-del-mundo. No necesitamos una visión sinóptica de algo llamado "el mundo". A lo sumo, lo que necesitamos es una narrativa sinóptica de cómo llegamos a hablar como lo hacemos. Debemos dejar de buscar una imagen unificada y un vocabulario maestro. Nos debemos limitar a asegurarnos de que no estamos cargados de formas de

${ }^{4}$ Ibid., p. 3.

${ }^{5}$ P. Pettit, "Existentialism, Quietism and Philosophy", p. 306.

${ }^{6}$ Ibid., p. 306. Pettit añade que "la filosofía hoy en día probablemente impone más retos y es más difícil de lo que nunca ha sido". Probablemente esto es verdad, pero se puede decir lo mismo de la teología cristiana.

Diánoia, vol. LI, no. 56 (mayo 2006). 
hablar obsoletas, y entonces asegurarnos de que aquellos vocabularios que todavía son útiles no se mezclen unos con otros.

Las narrativas que cuentan cómo nacieron estos diversos vocabularios nos ayudan a ver que las terminologías que empleamos para algunos propósitos no necesitan conectarse claramente con aquellas que empleamos para otros propósitos; que podemos simplemente dejar que dos prácticas lingüísticas coexistan pacíficamente, lado a lado. Esto es lo que Hume sugirió que hiciéramos con el vocabulario de predicción y con el de asignaciones de responsabilidad. La lección que los pragmatistas sacaron de Hume fue que los filósofos no deben rascarse cuando no tienen comezón. Cuando ya no existe una audiencia fuera de la disciplina que manifieste interés por un problema filosófico, ese problema debe verse con desconfianza.

Es posible que naturalistas como Pettit y Leiter respondan que están interesados en la verdad filosófica más que en satisfacer la última moda. Ésta es la misma estrategia retórica que usaron los aristotélicos del siglo XVII para rechazar a Hobbes y a Descartes. Hobbes respondió que aquellos que todavía seguían metidos en lo que llamó "la atmósfera enrarecida de la vana filosofía", estaban bajo el control de una terminología obsoleta que hacía ver como problemas apremiantes los que ellos discutían. Los quietistas contemporáneos piensan del mismo modo acerca de sus opositores activistas; creen que el vocabulario del representacionismo está tan gastado y es tan sospechoso como el del hilomorfismo.

Esta perspectiva antirrepresentacionista se puede encontrar en varias contribuciones a un libro reciente titulado Naturalism in Question, compilado por Mario de Caro y David Macarthur, y es más explícita en el ensayo de Huw Price, "Naturalism without Representationalism". Price hace una distinción muy útil entre naturalismo del objeto [object naturalism] y naturalismo del sujeto [subject naturalism]. El naturalismo del objeto es "el punto de vista de que en un sentido importante, todo lo que hay es el mundo estudiado por la ciencia". ${ }^{7}$ El naturalismo del sujeto, por otro lado, dice simplemente que "nosotros, los humanos, somos criaturas naturales, y si las afirmaciones y las ambiciones de la filosofía entran en conflicto con este punto de vista, entonces la filosofía debe ceder el paso".

Mientras que los naturalistas del objeto se preocupan por el lugar de las no partículas en un mundo de partículas, dice Price, los naturalistas del sujeto ven estos "problemas de localización" como "problemas acer-

${ }^{7}$ H. Price, "Naturalism without Representationalism", p. 73. 
ca de la conducta lingüística humana". ${ }^{8}$ Los naturalistas del objeto se preocupan por cómo es que las no partículas están relacionadas con las partículas porque, en palabras de Price, dan por sentado que "relaciones semánticas sustantivas 'palabra-mundo' son una parte de la mejor explicación científica de nuestro uso de los términos relevantes". 9 Los naturalistas del sujeto son deflacionistas semánticos: no ven la necesidad de tales relaciones y, en particular, de la de "hacer verdadero" (being made true by). Piensan que una vez que hemos explicado los usos de los términos relevantes, ya no hay ningún problema acerca de la relación de esos usos con el mundo.

Bjørn Ramberg, en un artículo llamado "Naturalizing Idealizations", usa "naturalismo pragmático" para designar el mismo acercamiento a los problemas filosóficos que Price llama "naturalismo del sujeto". Ramberg escribe:

La reducción, dice el pragmatista, es una metaherramienta de la ciencia; una manera de extender sistemáticamente el dominio de un conjunto de herramientas para manejar las tareas explicativas a las que se enfrentan los científicos. La naturalización, por contraste, es una meta de la filosofía: es la eliminación de los huecos metafísicos entre los rasgos característicos con los cuales tratamos con agentes y pensadores, por un lado, y los rasgos característicos con referencia a los cuales empíricamente generalizamos sobre las relaciones causales entre objetos y sucesos, por el otro. Es sólo en el contexto de cierta metafísica que la herramienta científica se vuelve una herramienta filosófica, un instrumento de ontología legislativa. ${ }^{10}$

El naturalismo pragmático, continúa Ramberg, "trata el hueco mismo, ése que transforma la reducción a un proyecto filosófico, como un síntoma de una disfunción en nuestro vocabulario filosófico". La cura para esta disfunción, en palabras de Ramberg, es dar "alternativas para lo que empieza a parecer como complejos conceptuales e ideas fijas" y explicar "cómo nuestra práctica podría cambiar si describiéramos las cosas $[. .$.$] con vocabularios modificados". { }^{11}$

El libro de Frank Jackson, From Metaphysics to Ethics, es un paradigma del naturalismo del objeto. Jackson dice que "la metafísica seria [...] continuamente se enfrenta al problema de la localización". La naturaleza de este problema se explica en el siguiente pasaje: "Dado que los

${ }^{8}$ Ibid., p. 76.

${ }^{9}$ Ibid., p. 78.

${ }^{10}$ Ramberg, "Naturalizing Idealizations: Pragmatism and the Interpretive Strategy", p. 43.

${ }^{11}$ Ibid., p. 47. 
ingredientes son limitados, algunas supuestas características del mundo no van a aparecer explícitamente en alguna descripción más básica [...]. Inevitablemente hay una gran cantidad de supuestas características de nuestro mundo que debemos o bien eliminar, o bien localizar." 12

Los naturalistas del sujeto, en cambio, no están interesados en el concepto de "características del mundo meramente supuestas", a menos que se entienda por éste algo así como "un tema del que no vale la pena hablar". Su pregunta no es "¿cuáles son las características que el mundo realmente tiene?", sino "¿qué temas vale la pena discutir?" Los naturalistas del sujeto pueden pensar que la cultura como un todo estaría mejor si cierto juego del lenguaje ya no se jugara, pero no argumentan que algunas de las palabras utilizadas en esa práctica hacen referencia a entidades irreales. Tampoco instan a que se entiendan algunos enunciados como algo completamente diferente de aquello a lo que supuestamente hacen referencia.

Para Jackson, el método de lo que llama "metafísica seria" es el análisis conceptual, por la siguiente razón:

La metafísica seria nos exige abordar los casos en los que asuntos descritos en un vocabulario se hacen verdaderos por asuntos descritos en otro vocabulario. Pero, ¿cómo podríamos abordar esta cuestión sin considerar cuándo es correcto describir un asunto en términos de los diversos vocabularios? [...] Y hacer eso [...] es hacer análisis conceptual. ${ }^{13}$

Pero el análisis conceptual no le dice al metafísico serio qué asuntos hacen verdaderos cuáles enunciados acerca de otros asuntos. Ya lo sabe. Como continúa diciendo Jackson, "no se le otorga al análisis conceptual un papel al determinar la naturaleza fundamental del mundo; más bien se le asigna un papel central al determinar qué decir en términos menos fundamentales dada una descripción del mundo expuesta en términos más fundamentales". ${ }^{14}$

Como ya he enfatizado, los naturalistas del sujeto no están interesados en el concepto clave de Jackson, el de "hacer verdadero". Se conforman, dice Price, con "una explicación del uso de términos semánticos, en tanto que no dicen nada de peso teórico acerca de si estos términos 'refieren' o 'tienen condiciones de verdad'". ${ }^{15}$ La tarea fundamental del naturalista del sujeto, continúa diciendo, es "dar cuenta de

${ }^{12}$ Frank Jackson, From Metaphysics to Ethics: A Defence of Conceptual Analysis, p. 5.

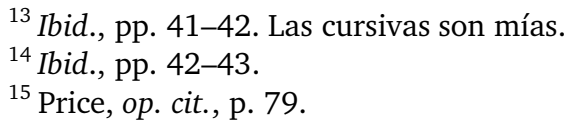

Diánoia, vol. LI, no. 56 (mayo 2006). 
los usos de los diversos términos - entre ellos, de los mismos términos semánticos- en las vidas de las criaturas naturales que están en un ambiente natural".

Si piensas que hay una relación como la de "hecho verdadero por", entonces puedes todavía tener esperanzas, como Jackson, de corregir las prácticas lingüísticas de tu tiempo sobre bases teóricas, más que simplemente en términos político-culturales, puesto que tu conocimiento $a$ priori de lo que hace verdaderos los enunciados te permite evaluar la relación entre la cultura de tu tiempo y la naturaleza intrínseca de la realidad misma. Pero naturalistas del sujeto como Price pueden criticar la cultura sólo argumentando que una cultura alternativa propuesta le ayudaría más a nuestros propósitos más amplios.

Price se enfrenta a Jackson con la siguiente pregunta: "[si podemos explicar] por qué las criaturas naturales en un ambiente natural llegan a hablar de estas formas plurales — de 'verdad', 'valor', 'significado', 'causalidad', etc.-, ¿qué pregunta queda? ¿Qué deuda tiene ahora la filosofía con la ciencia?"16 Esa pregunta se puede expandir en los siguientes términos: si sabes no sólo cómo se usan las palabras, sino también qué propósito tiene, o no tiene, usarlas así, ¿qué otra cosa podrías esperar que te dijera la filosofía?

Si quieres saber acerca de la relación entre el lenguaje y la realidad, continúa diciendo el quietista, considera cómo los primeros homínidos podrían haber empezado a usar señas y ruidos para coordinar sus acciones; luego consulta a los antropólogos y a los historiadores intelectuales. Ésta es la gente que te puede decir cómo nuestra especie progresó desde organizarse para buscar comida hasta construir ciudades y escribir libros. Dadas narrativas como éstas, ¿qué propósito tiene añadir una explicación de la relación de estos logros con la conducta de partículas físicas?

Tanto Jackson como Price se enorgullecen de ser naturalistas, pero cuando hablan de la "naturaleza", les vienen a la mente cosas diferentes. Cuando Jackson usa esa palabra piensa en partículas. Un naturalista del sujeto como Price piensa, en cambio, en organismos haciéndole frente a su medio ambiente y mejorándolo. El naturalista del objeto manifiesta su miedo a los fantasmas al insistir que todo concuerde, de alguna manera, con los movimientos de los átomos a través del vacío. El naturalista del sujeto manifiesta su miedo a los fantasmas al insistir en que nuestras historias acerca de cómo la evolución condujo de los protozoarios al Renacimiento no deben contener ninguna disconti-

$$
{ }^{16} \text { Ibid., p. } 87 .
$$


nuidad repentina; que sea una historia de complejidad gradualmente creciente de la estructura fisiológica que facilita una conducta cada vez más compleja.

Para el naturalista del sujeto, la importancia de la máxima de Price de que "somos criaturas naturales en un medio ambiente natural" es que debemos recelar de trazar líneas entre clases de organismos en términos no conductuales y no fisiológicos. Esto significa que no debemos usar términos como "intencionalidad", "conciencia" o "representación", a menos que podamos especificar, por lo menos de un modo aproximado, qué tipo de conducta es suficiente para mostrar la presencia de los referentes de estos términos.

Por ejemplo, si queremos decir que los calamares tienen intencionalidad, pero que los paramecios no, o que hay algo que es ser un murciélago, pero no hay nada que sea ser una lombriz, o que los insectos representan su medio ambiente mientras que las plantas meramente responden a él, debemos estar dispuestos a explicar cómo es que lo podemos decir, a especificar qué hechos conductuales o fisiológicos son relevantes para esta afirmación. Si no podemos hacerlo, estamos levantando polvo y luego quejándonos de que no vemos. Estamos inventando fantasmas para luego darles trabajo a los cazafantasmas.

Este énfasis en los criterios conductuales nos recuerda el verificacionismo de los positivistas; pero es diferente en que no es el producto de una teoría general acerca de la naturaleza del significado que nos permita distinguir el sentido del sinsentido. El naturalista del sujeto puede admitir alegremente que cualquier expresión tendrá sentido si le das uno. Sucede, más bien, que las distinciones filosóficas tradicionales complican gratuitamente las narrativas de la evolución biológica. En el mismo espíritu, los teólogos liberales argumentan que las cuestiones acerca del número de los sacramentos, a pesar de que son perfectamente inteligibles, nos distraen del mensaje cristiano.

Los católicos fundamentalistas, por supuesto, insisten en que tales cuestiones son todavía muy importantes. Los naturalistas del objeto son igualmente insistentes en que es importante preguntar, por ejemplo, cómo ciertas configuraciones de partículas físicas logran manifestar virtudes morales. Los cristianos quietistas piensan que las preguntas en las que insisten estos católicos son reliquias de un periodo relativamente primitivo en la recepción del mensaje de Jesucristo. Los filósofos quietistas piensan que las preguntas que todavía formulan sus colegas activistas eran suficientemente razonables en el siglo XVII, eran un producto predecible del choque producido por la Nueva Ciencia; sin embargo, ahora se han vuelto irrelevantes para la vida intelectual. La 
fe cristiana sin sacramentalismo y lo que Price llama "naturalismo sin representacionismo" son ambas iniciativas político-culturales.

Hasta ahora he estado exponiendo la oposición entre naturalistas del objeto y naturalistas del sujeto en términos muy generales. En lo que resta trataré de mostrar la relevancia de esta oposición para un par de controversias filosóficas actuales.

La primera de éstas es la discrepancia entre Timothy Williamson y John McDowell. La antología de Brian Leiter a la que antes me referí incluye un ensayo muy polémico de Williamson titulado "Past the Linguistic Turn?" Williamson empieza atacando la perspectiva que John McDowell toma de Hegel, Wittgenstein y Sellars; a saber: "Puesto que el mundo es todo lo que es el caso [...], no hay ningún hueco entre el pensamiento, como tal, y el mundo." Williamson parafrasea esto como la afirmación de que "lo conceptual no tiene límites externos más allá de los cuales resida la realidad no conceptualizada" y, de nuevo, como la tesis de que "cualquier objeto puede ser pensado". ${ }^{17}$

Williamson dice que:

a pesar de lo que McDowell ha mostrado, puede haber limitaciones necesarias en cualquier pensador posible. No sabemos si hay objetos elusivos. No queda claro qué motivaría la afirmación de que no hay ninguno, si no fuera una forma de idealismo. No debemos adoptar ninguna concepción de la filosofía que excluya objetos elusivos sobre bases metodológicas. ${ }^{18}$

Creo que McDowell, un autodeclarado quietista, podría responder diciendo que de hecho deberíamos adoptar una concepción de la filosofía que excluya objetos elusivos. Deberíamos hacerlo por razones de política cultural. Deberíamos decir que las culturas que se preocupan acerca de preguntas incontestables como " ¿hay limitaciones necesarias sobre todo posible pensador?", “ipodría Dios cambiar las verdades de la aritmética?", “¿estoy soñando ahora?” y “iestá mi espectro de color invertido con respecto al tuyo?" son menos avanzadas que aquellas que respetan la máxima pragmática de Peirce. A las culturas superiores no les interesa lo que Peirce llamó "dudas imaginarias" [make-believe doubts].

Williamson está equivocado al sugerir que sólo el idealismo podría motivar la tesis de McDowell. La diferencia entre el idealismo y el pragmatismo es la que existe entre los argumentos metafísicos o epistemoló-

${ }^{17}$ Williamson, "Past the Linguistic Turn?", p. 109.

${ }^{18}$ Ibid., p. 110.

Diánoia, vol. LI, no. 56 (mayo 2006). 
gicos en favor de la afirmación de que cualquier objeto puede ser pensado y los argumentos político-culturales en favor de esa afirmación. Los pragmatistas piensan que la idea de limitaciones necesarias sobre cualquier pensador posible es tan extraña como la tesis agustiniana acerca de la inevitabilidad del pecado: non posse non peccare. Ninguna puede ser refutada, pero la salud mental pide que ambas sean descartadas de entrada. ${ }^{19}$

El choque de opiniones entre McDowell y Williamson es el epítome de la oposición entre dos líneas de pensamiento recientes dentro de la filosofía analítica. Una va de Wittgenstein, pasando por Sellars y Davidson, a McDowell y Brandom. La otra está asociada con lo que Williamson llama "el resurgimiento de la teorización metafísica, de espíritu realista [...] asociada con Saul Kripke, David Lewis, Kit Fine, Peter van Inwagen, David Armstrong y muchos otros". ${ }^{20}$ La meta de estos intentos es ir más allá del giro lingüístico, dice Williamson, "para descubrir qué clase fundamental de cosas hay, y qué propiedades y relaciones tienen, no cómo las representamos". ${ }^{21} \mathrm{El}$ contraste entre estas dos líneas de pensamiento será más vívido para cualquiera que vaya de una a otra de las dos colecciones de artículos de las que he estado citando: The Future for Philosophy de Leiter y Naturalism in Question de De Caro y Macarthur.

Los quietistas piensan que ninguna clase de cosa es más fundamental que otra. El hecho de que, tal como lo pone Jackson, no puedas cambiar nada sin cambiar los movimientos o las posiciones de partículas físicas elementales no muestra que haya un problema acerca de cómo estas partículas dan cabida a las no partículas. No está más cargado filosóficamente que el hecho de que no te puedes meter con las partículas sin simultáneamente meterte con muchas otras cosas. Expresiones como "la naturaleza de la realidad" o "el mundo como realmente es", admite el quietista, han tenido en el pasado un papel en la producción de cambios culturales deseables. Pero también lo han tenido muchas otras escaleras que sería aconsejable que tiráramos.

${ }^{19}$ El pragmatismo adopta su posición en contra de todas las doctrinas que sostienen, en palabras de Leo Strauss, que "incluso probando que cierta opinión es indispensable para vivir bien, simplemente se prueba que la opinión en cuestión es un mito beneficioso: no se prueba que sea verdadero" (Natural Right and History, p. 6). Strauss continúa diciendo que "la utilidad y la verdad son dos cosas completamente diferentes". Los pragmatistas no piensan que sean lo mismo, pero sí piensan que no puedes tener la segunda sin la primera.

${ }^{20}$ Williamson, op.cit., p. 111.

${ }^{21}$ Ibid., pp. 110-111.

Diánoia, vol. LI, no. 56 (mayo 2006). 
Los quietistas que no se interesan por el concepto de "cómo es el mundo independientemente de nuestras formas de representarlo" se mostrarán reacios ante la tesis de Williamson de que "lo que hay determina lo que para nosotros tiene significado". Pero también se mostrarán reacios ante la afirmación de los idealistas de que nuestros significados determinan lo que hay. Los quietistas quieren ir más allá del realismo y del idealismo al dejar de contrastar un mundo representado con nuestras formas de representarlo. Esto significa renunciar al concepto de representaciones lingüísticas del mundo excepto en la medida en que pueda reconstruirse dentro de una semántica inferencialista. Dicha semántica renuncia a lo que Price llama "relaciones palabra-mundo sustanciales" a favor de descripciones de la interacción de organismos que usan el lenguaje con otros organismos semejantes y con su medio ambiente.

La controversia acerca de la semántica inferencialista es la segunda que quiero discutir brevemente. La objeción mejor conocida al inferencialismo de Brandom es la de Fodor. El enfrentamiento entre Fodor y Brandom es el arquetipo, no sólo de la diferencia entre la semántica representacionista y la inferencialista, sino del conflicto más grande entre atomistas y holistas al que me referí antes. Fodor piensa que la filosofía puede unirse con la ciencia cognitiva para averiguar cómo funcionan los mecanismos de la mente y del lenguaje. Brandom es escéptico acerca de la idea de que haya tales mecanismos.

Brandom lleva el holismo davidsoniano hasta sus límites. Tal como Davidson lo hizo en "A Nice Derangement of Epitaphs", repudia la idea de que haya algo así como "un lenguaje", algo que se divide en partes llamadas "significados" o "representaciones lingüísticas" que pueden luego correlacionarse con parte del mundo físico. Trata de realizar la esperanza de Quine y Davidson en —según lo ha puesto Kenneth Taylor- "una teoría del significado en la que los significados no tienen ningún papel". ${ }^{22}$ Así es que abandona el concepto de que un enunciado tenga un "contenido cognitivo" que permanece constante en todas las aseveraciones en las que es usado. Brandom alegremente se desliza en lo que Fodor burlonamente describe como "una bien engrasada y muy

${ }^{22}$ Kenneth Taylor, Truth and Meaning: An Introduction to the Philosophy of Language, p. 147. Taylor piensa que la aversión de Davidson por los significados es un resultado de su preferencia por los lenguajes extensionales. Esto pudo haber influido en el pensamiento (temprano) de Davidson, pero no en el de Brandom. Una vez que nos deshacemos de la relación de "hacer verdad", no habrá ninguna razón para pensar en lenguajes extensionales sospechosos. 
usada pendiente resbaladiza", al final de la cual está la opinión de que "dos personas nunca quieren decir la misma cosa con lo que dicen". ${ }^{23}$

Brandom hace esto porque quiere desechar la idea de que yo pongo lo que está en mi cabeza - un contenido cognitivo, un candidato para una representación exacta de la realidad- en la tuya haciendo ruidos que llevan a cabo esta transmisión. Brandom espera reemplazarla con una teoría de lo que llama "marcador doxástico": llevando la cuenta de los compromisos de nuestros interlocutores para realizar ciertas acciones en ciertas condiciones (incluyendo el asentimiento o el disentimiento a ciertas aseveraciones).

Dichos compromisos se atribuyen por referencia a normas sociales. Estas normas nos autorizan a reclamarle a gente que, habiendo dicho "Te prometo que te voy a pagar" o "Me uniré a la cacería", no hacen nada en ese sentido. Lo mismo vale para gente que, habiendo expresado " $p$ " y "si $p$ entonces $q$ ", se rehúsan obstinadamente a asentir a " $q$ ". A diferencia de los animales, nosotros podemos jugar lo que Brandom llama el "juego de dar y pedir razones". Nuestra habilidad para jugar este juego es lo que nos posibilita suponer un dominio sobre los otros animales. Decir que nosotros, a diferencia de los animales, tenemos mentes es sólo otra forma de decir que nosotros, pero no ellos, jugamos ese juego. Fodor, por el contrario, al averiguar cómo funciona el cerebro, no nos ayudará a averiguar cómo funciona la mente, ${ }^{24}$ puesto que la mente no es un aparato de representaciones, sino más bien un conjunto de prácticas sociales regidas por normas.

Brandom no se llama a sí mismo un "naturalista", tal vez porque piensa que el término podría cederse a los fanáticos de las partículas elementales. Pero el único propósito de su intento de reemplazar una semántica representacionista con una inferencialista es el de contar una historia acerca de la evolución cultural —la evolución de prácticas sociales (y, en particular, lingüísticas) - que se enfoca en cómo estas prácticas le dieron a nuestros ancestros una ventaja evolutiva. A menos que uno esté convencido de que las partículas de alguna manera tienen un estatus ontológico superior al de los organismos, esa historia no podrá parecer más naturalista.

Brandom admite alegremente que "Una palabra - 'perro', 'estúpido', 'republicano' - tiene un significado diferente en mi boca que en la tuya, en la medida en que lo que se sigue de su posibilidad de aplicación -las consecuencias de su aplicación- es diferente para mí, en virtud

\footnotetext{
${ }^{23}$ Jerry Fodor, “Why Meaning (Probably) Isn't Conceptual Role”, p. 143.

${ }^{24} \mathrm{He}$ argumentado más detalladamente en este tenor en "The Brain as Hardware, Culture as Software".
} 
de mis creencias colaterales diferentes." 25 Pero esta diferencia no es un problema para nadie, excepto para filósofos que, como Fodor, toman seriamente el concepto de "contenido cognitivo".

Es muy posible que busquemos relaciones sustantivas palabra-mundo en tanto que formulemos preguntas fregeanas acerca de pequeños átomos de significado lingüístico, como "¿La aseveración de que la estrella de la mañana es la estrella de la tarde tiene el mismo contenido cognitivo que la aseveración de que la cosa que llamamos estrella de la mañana es la misma cosa que la que llamamos la estrella de la tarde?" Si "mismo contenido cognitivo" sólo quiere decir "será igualmente bueno para la mayoría de los propósitos", entonces la respuesta es sí. Pero los fregeanos, invocando la prueba de traducción de Church, hacen caso omiso del hecho de que cualquiera de estos enunciados comúnmente sirve para este fin. La pregunta real, dicen, no es acerca de los usos, sino acerca de los sentidos, significados e intensiones. El sentido, dicen estos filósofos, determina la referencia en la misma forma en que las señales sobre el mapa determinan qué pedazo de la realidad mapea el mapa. Los significados no pueden ser lo mismo que los usos, puesto que hay una diferencia entre la semántica y la pragmática. Es la semántica la que determina la igualdad y la diferencia del contenido cognitivo.

Tendríamos algún uso para el concepto de "mismo contenido cognitivo" sólo si tratáramos de mantener creencia y significado separados, como pensó Frege que deberíamos hacer y como nos dijo Quine que no deberíamos hacer. Si continuamos por el camino que despejaron Quine y Davidson, llegaremos a estar de acuerdo con Brandom en que "fenómenos lingüísticos particulares ya no pueden distinguirse como 'pragmáticos' o ‘semánticos'”. ${ }^{26}$ Una distinción entre estas dos disciplinas no le es más útil a Brandom de lo que la distinción entre saber una lengua y manejarnos en el mundo le fue a Davidson.

Hasta aquí las dos controversias sobre las que quería comentar. Espero que mi discusión de los desacuerdos entre McDowell y Williamson y entre Brandom y Fodor haya ayudado a esclarecer por qué pienso que es tan útil la distinción de Price entre dos formas de naturalismo. Los naturalistas del sujeto, como Price, Ramberg y yo, exhortamos a nuestros colegas activistas a que dejen de hablar acerca de grandes cosas como Experiencia o Lenguaje, las entidades fantasmas que Locke, Kant y Frege inventaron para reemplazar a la Realidad como el tema de la

${ }^{25}$ Robert Brandom, Making It Explicit, p. 587.

${ }^{26}$ Ibid., p. 592. 
filosofía. Proceder de este modo podría conducir, a la larga, a evacuar las así llamadas "áreas centrales" de la filosofía. Los naturalistas del objeto como Jackson, Leiter, Pettit y Fodor temen que la filosofía no sobreviva si se purgara a sí misma de este modo. Pero los naturalistas del sujeto sospechan que la única cosa que nuestra disciplina perdería sería su insularidad.

[Traducción de Gustavo Ortiz-Millán]

\section{BIBLIOGRAFÍA}

Anscombe, G.E.M., "Modern Moral Philosophy", Ethics, Religion and Politics, University of Minnesota Press, Minneapolis, 1981, pp. 26-42.

Brandom, Robert, Making It Explicit, Harvard University Press, Cambridge, Mass., 1994. [Versión en castellano: Hacerlo explícito. Razonamiento, representación y compromiso discursivo, trad. Ángela Ackermann y Joana Rosselló, Herder, Barcelona, 2005.]

De Caro, Mario y David Macarthur (comps.), Naturalism in Question, Harvard University Press, Cambridge, Mass., 2004.

Fodor, Jerry, "Why Meaning (Probably) Isn't Conceptual Role", en Stephen Stich y Ted Warfield (comps.), Mental Representations, Blackwell, Oxford, 1994, pp. 142-156.

Jackson, Frank, From Metaphysics to Ethics: A Defence of Conceptual Analysis, Oxford University Press, Oxford, 1998.

Leiter, Brian (comp.), The Future for Philosophy, Oxford University Press, Oxford, 2004.

Pettit, Philip, "Existentialism, Quietism and Philosophy", en Leiter (comp.), The Future for Philosophy, pp. 304-327.

Price, Huw, "Naturalism without Representationalism", en De Caro y Macarthur (comps.), Naturalism in Question, pp. 71-88.

Ramberg, Bjørn, "Naturalizing Idealizations: Pragmatism and the Interpretive Strategy", Contemporary Pragmatism, vol. 1, no. 2, 2004, pp. 1-63.

Rorty, Richard, "The Brain as Hardware, Culture as Software", Inquiry, vol. 47, no. 3, 2004, pp. 219-235.

Strauss, Leo, Natural Right and History, The University of Chicago Press, Chicago, 1968.

Taylor, Kenneth, Truth and Meaning: An Introduction to the Philosophy of Language, Blackwell, Oxford, 1998.

Williamson, Timothy, "Past the Linguistic Turn?", en Leiter (comp.), The Future for Philosophy, pp. 106-128.

Recibido el 10 de febrero de 2006; aceptado el 23 de febrero de 2006.

Diánoia, vol. LI, no. 56 (mayo 2006). 